\title{
Structural and Optical Characteristics of Silica Nano- tubes Using CNTs as Template
}

\section{Rasoul Malekfar ${ }^{1, *}$, M. Hassan Rajabi² and M. Hossein Majless Ara $^{3}$}

Silica coated multi-wall carbon nanotubes (MWCNTs), silica@MWCNTs and nanocomposites were synthesized by a sol-gel method. By using the synthesized nanocomposites and also CNTs as templates, silica nanotubes (silica-NTs) were prepared. The optical properties of fabricated nanocomposites and nanotubes were characterized by back-scattering micro Raman, UV/Vis/NIR and FT-IR spectra, which show the presence of CNTs structure in the nanocomposites. UV/Vis/NIR and FT-IR spectra also show the presence of silica compounds. The recorded spectra from UV/Vis/NIR and FT-IR also confirm the presence of silica compounds in the nanotubes. The results of FE-SEM imaging data indicate that the synthesized samples are MWCNTs coated uniformly by silica molecules, which act as the template to synthesize silica-NTs.

Keywords: Carbon nanotubes (MWCNTs); Silica; Raman; Ultra violet/visible/near infrared (UV/Vis/NIR)

Citation: Rasoul Malekfar, M. Hassan Rajabi and M. Hossein Majless Ara "Structural and Optical Characteristics of Silica Nanotubes Using CNTs as Template”, Nano-Micro Lett. 2, 268-271 (2010). doi:10.3786/nml.v2i4.p268-271

Since the discovery of carbon nanotubes (CNTs) by Iijima, which nowadays play an important role in different fields of nanotechnology, their optical, electrical, thermal and mechanical properties have been widely studied. There are different methods to use CNTs properties to synthesize new structures. These include the synthesis of various CNTs composites with metals, ceramics and polymers.

The coating process of CNTs with matrix can eliminate some of the problems which can be appeared in the nanocomposites synthesis. These problems include non-uniform dispersion of CNTs in the final product and weak adhesion between the nanocomposite precursor structures.

Recently, a lot of progress has been made in the field of binding different insulating materials with CNTs e.g. for manufacturing field effect transistors, gas sensors, molecular circuits, switches, etc. [1]. Silica is an insulator and when CNTs is being coated with it, toughness [2], thermal conductivity [3], and nonlinear optical properties can be modified and enhanced [4]. One of the main applications of CNTs is to use them as templates to synthesize other nano-structures such as nanotubes, nanowires and nanorods. In the present paper, we have mainly concentrated to report the fabrication and characterization procedures to synthesize silica-NTs.

Silica coated MWCNTs (silica@MWCNTs) have been proved to be a super-dielectric with a very large low-frequency dielectric coefficient and low dielectric loss [5]. Different methods such as liquid phase deposition [6], electrodeposition [7], sol-gel methods [8], reverse micelle [9], and plasmaenhanced chemical vapor deposition [10], have been successfully applied to synthesize silica@MWCNTs.

In this paper, by using sol-gel method, silica@MWCNTs was synthesized [11-15]. By calcination processes of the synthesized nanocomposites, silica-NTs, have been fabricated. The prepared silica-NTs are hydrophilic, biocompatible and photoluminescent. This nanostructure has many applications in bioseparation, biocatalysis, biosensors, drug/gene delivery carriers and optoelectronic nanodevices [16].

In this paper, we report the synthesis procedure of silica@MWCNTs and silica-NTs, and the structure, morphology and size determinations of the produced nanostructures using

1Physics Department, Faculty of Basic Sciences, Tarbiat Modares University, Tehran P.O. Box 14115-175, I.R. Iran, Email: Malekfar@Modares.ac.ir 2Department of Physics, Faculty of Basic Sciences, Member of Young Researches Club, Islamic Azad University, North Tehran Branch 
dispersive back-scattering micro-Raman, Ultra Violet/Visible/ Near Infrared (UV/Vis/NIR), Fourier Transform Infrared spectroscopy (FT-IR), Field Emission Scanning Electron Microscopy (FE-SEM), and X-ray Diffraction (XRD).

\section{Experimental procedure}

\section{Materials}

MWCNTs, 93\% purity, with diameters ranging between 30 and $50 \mathrm{~nm}$ and lengths ranging from 5 to $15 \mu \mathrm{m}$, were produced in our laboratory by catalytic chemical vapor deposition (CCVD) method [17]. Cetyltrimethylammonium bromide (CTAB), 3aminopropyltriethanoxysilane (APTES), ammoniumhy droxid (25\%) and tetraethoxysilane (98\%) were all acquired from Aldrich.

\section{Preparation of silica@MWCNTs and silica-NTs}

Silica@MWCNTs were prepared by hydrolysis of tetraethyloxy-silane (TEOS) in the presence of MWCNTs. MWCNTs (170 mg), obtained by CCVD method (93\% purity) without further purification, were ultrasonically dispersed into a fixed-volume mixture of $\mathrm{H}_{2} \mathrm{O}(25 \mathrm{ml})$, CTAB (50 mg) (as surfactant) and $0.25 \mathrm{ml}$ APTES (as coupling agent) were mixed for $20 \mathrm{~min}$ at $40^{\circ} \mathrm{C}$, and then the mixture was stirred for another 3 h. Afterward, a mixture of $2.5 \mathrm{ml}$ of TEOS with $2 \mathrm{ml}$ of $\mathrm{H}_{2} \mathrm{O}, 25$ $\mathrm{ml}$ of ethanol and $1.6 \mathrm{ml}$ of $\mathrm{NH}_{4} \mathrm{OH}(25 \%)$ was added and the whole mixture was sonicated for 60 min under $40^{\circ} \mathrm{C}$, and then stirred for $1 \mathrm{~h}$. After the sonication procedures, the mixture was filtered in order to remove free silica particles and then washed with distilled water and absolute alcohol for at least four times. Finally, the products were dried in open atmosphere yielding silica@MWCNTs. Then silica@MWCNTs was oxidized in a furnace at $600^{\circ} \mathrm{C}$ for $8 \mathrm{~h}$ to remove the CNTs templates and then silica-NTs were acquired.

\section{Results and discussion}

Figure 1 shows the recorded absorbance UV/Vis/NIR spectra of the three products of pure MWCNTs, silica@ MWCNTs and silica-NTs. The MWCNTs spectrum has a clear, broad strong peak at around $270 \mathrm{~nm}$ [18]. The absorbance of the silica@MWCNTs has decreased at most of the spectral region.

However, the final product, i.e. silica-NTs, has shown lower absorbance in the entire spectral region, compared with MWCNTs and silica@MWCNTs. It is quite obvious that the

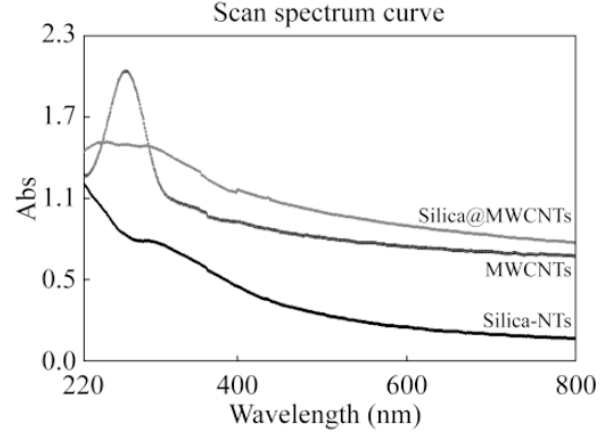

FIG. 1. UV/Vis/NIR spectra of MWCNTs, silica@MWCNTs and silica-NTs.

optical properties of MWCNTs have been changed after coating by silica.

In the silica-NTs, a weak absorbance peak in the UV frequency region still exists, confirming that the silica-NTs are transparent. The overall results of the recorded UV/Vis/NIR spectra of the synthesized samples indicate that in the silica@MWCNTs and silica-NTs the silica structure is clearly dominant [19].

Typical FT-IR spectra of the synthesized samples of silica@MWCNTs and silica-NTs are shown in Fig. 2. For silica@MWCNTs, the strong band situated at $1084 \mathrm{~cm}^{-1}$ corresponds to $\mathrm{Si}-\mathrm{O}-\mathrm{Si}$ asymmetric stretching vibration. However, two peaks observed at 811 and $948 \mathrm{~cm}^{-1}$ are attributed to Si-O-Si symmetric stretching vibration and $\mathrm{Si}-\mathrm{OH}$ stretching, respectively. The C-H stretching vibration is observed at 2982 $\mathrm{cm}^{-1}$. Moreover, two bands with central frequencies at 3425 and $1630 \mathrm{~cm}^{-1}$ are observed, which are attributed to the stretching and bending modes of $\mathrm{O}-\mathrm{H}$, respectively.

In the silica-NTs, the $\mathrm{Si}-\mathrm{O}-\mathrm{Si}$ asymmetric and symmetric stretching vibrations are clearly observed at 1094 and $817 \mathrm{~cm}^{-1}$, respectively. And also the $\mathrm{Si}-\mathrm{OH}$ stretching mode is centered at $957 \mathrm{~cm}^{-1}$. These results can confirm that silica has been uniformly coated by MWCNTs and also the synthesized silica-NTs lack carbon structure [20].

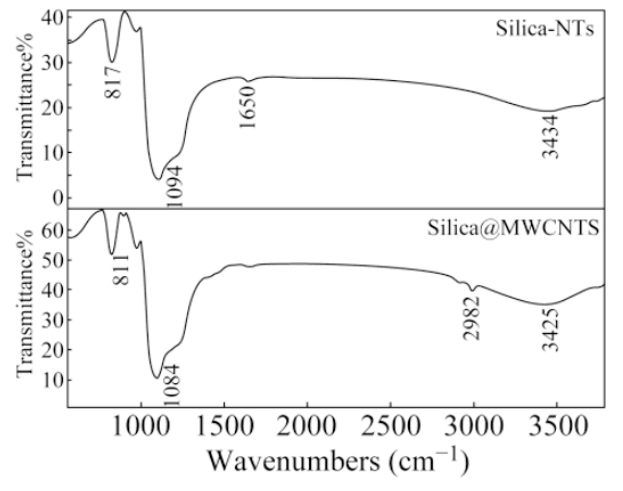

FIG. 2. FT-IR spectra of silica@MWCNTs and silica-NTs. 


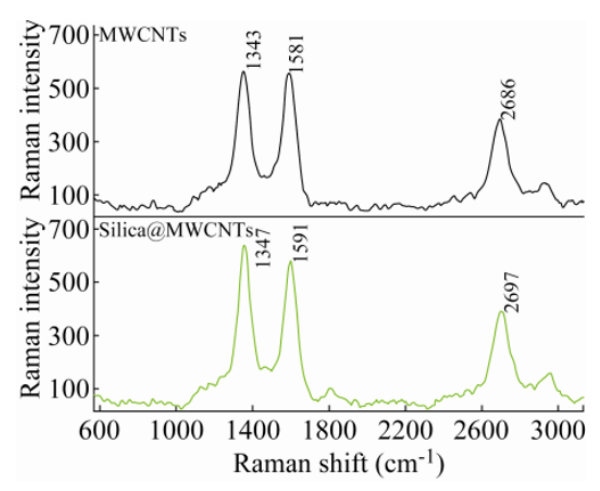

FIG. 3. Raman spectra of MWCNTs and silica@MWCNTs.

In the Raman spectra three strong peaks in the spectrum of silica@MWCNTs, shown in Fig. 3, are presented. The peaks at 1347 and $1591 \mathrm{~cm}^{-1}$ are assigned to the disorder-induced phonon and G-line tangential modes in CNTs, respectively. A peak situated at around $2697 \mathrm{~cm}^{-1}$, is attributed to $\mathrm{G}^{\prime}$ mode, as the overtone of $1347 \mathrm{~cm}^{-1}$. The $1591 \mathrm{~cm}^{-1}$ mode is lower in intensity in compare with the $1347 \mathrm{~cm}^{-1}$ mode, indicating that the synthesized MWCNTs, by CCVD method, have a low degree of graphitization and in the silica@MWCNTs the structure has not changed.

The FE-SEM image, shown in Fig. 4(a), clearly confirms that MWCNTs have been smoothly coated by silica with a wall thickness of the silica changing from 8 to $15 \mathrm{~nm}$. In the FE-SEM photograph shown in Fig. 4(b), it is clear that CNTs have played as the template for silica-NTs with a diameter of silica-NTs ranging between 40 to $55 \mathrm{~nm}$.

Figure 5 shows the XRD patterns of the silica@MWCNTs and silica-NTs. In the silica@MWCNTs, diffraction peaks at around $25.6^{\circ}, 27^{\circ}, 32.6^{\circ}, 48.5^{\circ}, 52.0^{\circ}$ and $67.6^{\circ}$ are assigned to the (400), (041), (510), (730), (731) and (790), respectively, and are readily recognized from XRD patterns. The observed diffraction peaks agree well with the structure of silicon oxide

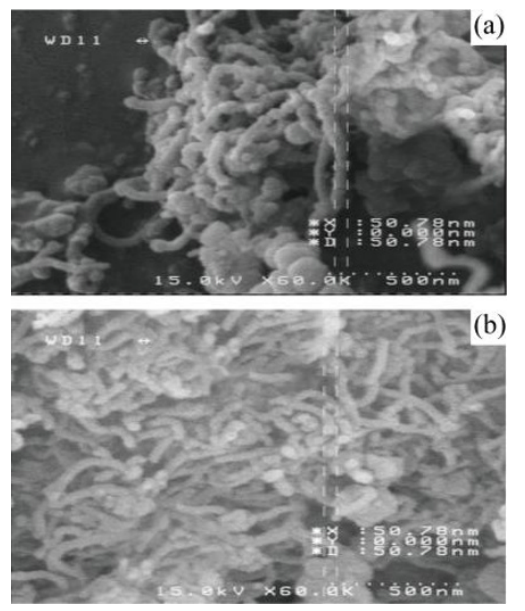

FIG. 4. FE-SEM microphotographs of (a) silica@MWCNTs; and (b) silica-NTs.
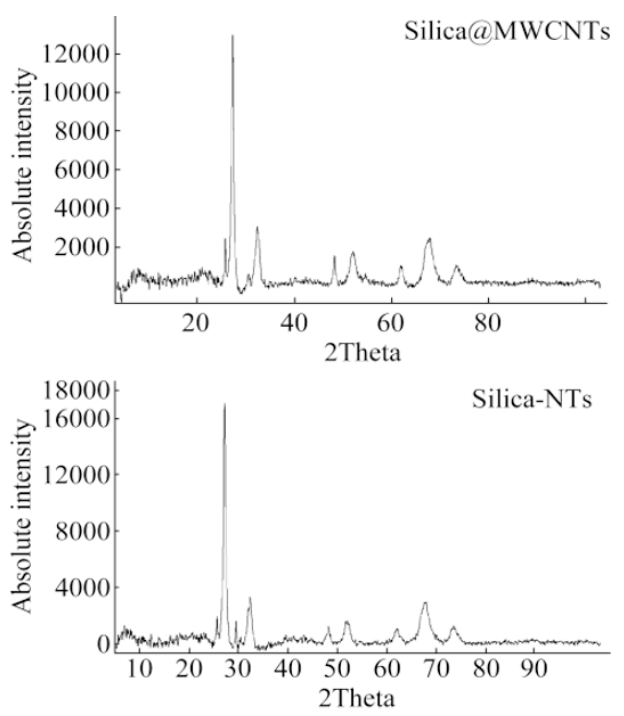

FIG. 5. XRD patterns of silica@MWCNTs prepared by the sol-gel process and silica-NTs.

diethyl amine (1999. JCPDS file No. 45-0569). It is clear that no other phases are detectable in the synthesized sample. For the silica-NTs, plane structures of (110), (011), (102), (113), (220) and $(223)$ are related to $25.8^{\circ}, 26.7^{\circ}, 32.3^{\circ}, 48.4^{\circ}, 53.0^{\circ}$ and $68.6^{\circ}$. These results confirm that the synthesized silica-NTs samples hold the hexagonal structure of silicon quartz alpha (1999. JCPDS file No. 85-1054).

\section{Conclusions}

The process of silica uniformly coated MWCNTs has been reported. In order to coat MWCNTs uniformly with silica we have used CTAB, as surfactant, and APTES, as coupling agent. In the following experimental procedure and by using the CNTs template present in the synthesized silica@MWCNTs, silica-NTs were produced. The results of the recorded back-scattering micro-Raman, UV/Vis/NIR and FT-IR spectroscopes clearly confirm that in the synthesized silica@MWCNTs, the MWCNTs structure has maintain its original structure. The FE-SEM images indicate that silica has been uniformly coated the MWCNTs. For the synthesized silica-NTs, UV/Vis/NIR, FT-IR spectra and FE-SEM images confirm that silica structure exists and CNTs have acted as the template for silica-NTs synthesis. The diameters of the produced silica-NTs are in the range of 30-50 $\mathrm{nm}$. By considering the XRD patterns, it has been confirmed that the synthesized structures of silica@MWCNTs and silica-NTs have similar structures as silicon oxide diethyl amine and silicon quartz alpha, respectively.

Received 15 October 2010; accepted 1 December 2010; published online 18 December 2010. 


\section{References}

1. H. Postma, T. Teepen, Z. Yao, M. Grifoni and C. Dekker, Science 293, 76 (2001). doi:10.1126/science.1061797

2. M. Olek, K. Kempa, S. Jurga and M. Giersig, Langmuir 21, 3146 (2005). doi:10.1021/1a0470784

3. H. Zhan, C. Zheng, W. Chen and M. Wang, Chem. Phys. Lett. 411, 373 (2005). doi:10.1016/j.cplett.2005.06.058

4. J. Ning, J. Zhang, Y. Pan and J. Guo, Mater. Sci. Lett. 22, 1019 (2003). doi:10.1023/A:1024745526978

5. T. Kempa, D. Carnahan, M. Olek, M. Correa, M. Giersig and M. Cross, J. Appl. Phys. 98, 34310 (2005). doi:10. $\underline{1063 / 1.1996836}$

6. E. Whitsitt and A. Barron, Nano Lett. 3, 775 (2003). doi:10.1021/n1034186m

7. M. Kanungo, H. Isaacs and S. Wong, Phys. Chem. 111, 17730 (2007).

8. Y. Liu, J. Tang, X. Chen, R. Wang, G. Pang, Y. Zhang and J. Xin, Carbon 44, 165 (2006). doi:10.1016/j.carbon. $\underline{2005.08 .020}$

9. M. Bottini, L. Tautz, H. Huynh, E. Monosov, N. Bottini, M. I. Dawson, S. Bellucci and T. Mustelin, Chem. Commun. 6, 758 (2005). doi:10.1039/b412876a

10. J. Hu, Z. Wang, W. Zhang, Z. Xu, Y. Wu, Z. Zhu and X. Duan, Carbon 44, 1581 (2006). doi:10.1016/j.carbon. $\underline{2006.01 .028}$
11. M. Kim, J. Hong, J. Lee, C. K. Hong and S. E. Shim, J. Colloid. Interface Sci. 322, 321 (2008). doi:10.1016/j.jcis. 2008.03.045

12. S. J. Guo, S. J. Dong and E. K. Wang, J. Phys. Chem. C 112, 2389 (2008). doi:10.1021/jp0772629

13. K. L. Ding, B. J. Hu, Y. Xie, G. M. An, R. T. Tao and H. Y. Zhang, J. Mater. Chem. 19, 3725 (2009). doi:10.1039/ b821386k

14. M. Zhang, Y. P. Wu, X. Z. Feng, X. W. He, L. X. Chen and Y. K. Zhang, J. Mater. Chem. 20, 5835 (2010). doi:10.1039/b925137e

15. M. Zhang, X. H. Zhang, X. W. He, L. X. Chen and Y. K. Zhang, Mater. Lett. 64, 1383 (2010). doi:10.1016/j.mat $\underline{\text { let.2010.03.032 }}$

16. M. Llusar and C. Sanchez, Chem. Mater. 20, 782 (2008). doi:10.1021/cm702141e

17. R. Malekfar and H. Asadi, AIP Conference Series. 935, 114 (2007).

18. L. Jiang, L. Gao and J. Sun, J. Colloid Inter. Sci. 260, 89 (2003).

19. S. O. Obare, Optical sensing strategies for the develop ment of novel chemical probes, PhD thesis, University of South Carolina, (2002).

20. C. Zheng, M. Feng, Y. Du and H. Zhan, Carbon 47, 2889 (2009). doi:10.1016/j.carbon.2009.02.004 